\title{
Sarcopenia Is Associated With a Risk of Mortality in People With Type 2 Diabetes Mellitus
}

OPEN ACCESS

Edited by:

Alok Raghav,

Ganesh Shankar Vidyarthi Memorial

Medical College, India

Reviewed by:

Kitti Amresh Gautam,

Ganesh Shankar Vidyarthi Memorial

Medical College, India

Jamal Ahmad,

Aligarh Muslim University, India

*Correspondence:

Yoshitaka Hashimoto

y-hashi@koto.kpu-m.ac.jp

${ }^{\text {t}}$ These authors have contributed equally to this work

Specialty section: This article was submitted to Clinical Diabetes,

a section of the journal

Frontiers in Endocrinology

Received: 26 September 2021 Accepted: 25 October 2021 Published: 11 November 2021

Citation:

Takahashi F, Hashimoto Y, Kaji A, Sakai R, Okamura T, Kitagawa N, Okada H, Nakanishi N, Majima S, Senmaru T, Ushigome E, Hamaguchi M, Asano M, Yamazaki M and Fukui M (2021) Sarcopenia Is Associated With a Risk of Mortality in People With Type 2 Diabetes Mellitus.

Front. Endocrinol. 12:783363. doi: 10.3389/fendo.2021.783363
Fuyuko Takahashi ${ }^{1 \dagger}$, Yoshitaka Hashimoto ${ }^{1 * t}$, Ayumi Kaji ${ }^{1}$, Ryosuke Sakai ${ }^{1}$, Takuro Okamura ${ }^{1}$, Noriyuki Kitagawa ${ }^{1,2}$, Hiroshi Okada ${ }^{1,3}$, Naoko Nakanishi ${ }^{1}$, Saori Majima ${ }^{1}$, Takafumi Senmaru ${ }^{1}$, Emi Ushigome ${ }^{1}$, Masahide Hamaguchi ${ }^{1}$, Mai Asano ${ }^{1}$, Masahiro Yamazaki ${ }^{1}$ and Michiaki Fukui ${ }^{1}$

1 Department of Endocrinology and Metabolism, Kyoto Prefectural University of Medicine, Graduate School of Medical Science, Kyoto, Japan, ${ }^{2}$ Department of Diabetology, Kameoka Municipal Hospital, Kameoka, Japan, ${ }^{3}$ Department of Diabetes and Endocrinology, Matsushita Memorial Hospital, Moriguchi, Japan

Background: Sarcopenia has reportedly been associated with increased risk of mortality in general populations. However, few studies have investigated the association between sarcopenia and mortality in older people with type 2 diabetes mellitus (T2D). This study aimed to investigate the effect of sarcopenia on incident all-cause mortality in older people with T2D.

Methods: Low muscle strength were set at handgrip strength $<28 \mathrm{~kg}$ for men and $<18 \mathrm{~kg}$ for women, and low skeletal muscle mass index (SMI), evaluated using the impedance body composition analyzer, were set at SMl $<7.0 \mathrm{~kg} / \mathrm{m}^{2}$ for men and $<5.7 \mathrm{~kg} / \mathrm{m}^{2}$ for women. People who had both low muscle strength and low SMI were diagnosed with sarcopenia. Due to a low incidence of all-cause mortality, the propensity score was used. The propensity score was evaluated using multivariable logistic regression models with the following parameters: age, sex, duration of diabetes, history of heart disease, history of cancer, smoking, exercise, alcohol, sodium-glucose cotransporter-2 inhibitor, glucagonlike peptide-1 receptor agonist, insulin, corticosteroid, hypertension, body mass index, glycosylated hemoglobin A1c, triglycerides, and creatinine, and the C-statistic was 0.89.

Results: In this prospective cohort study, 396 people with an average age and duration of diabetes of 71.3 (6.3) years and 16.3 (11.3) years, respectively, were included. Of those included, 14.6\% had sarcopenia. During the average 40.5 (16.5) months of follow-up, 13 people (6 out of the 338 without sarcopenia and 7 out of the 58 with sarcopenia) died. Incident rate were 5.1/1000 person years of follow-up in people without sarcopenia and 41.3/1000 person years of follow-up in people with sarcopenia. According to Cox regression analysis, sarcopenia was associated with all-cause mortality (adjusted hazard ratio: 6.12 , 95\% confidence interval: $1.52-24.7, p=0.011$ ).

Conclusion: Sarcopenia is associated with incident all-cause mortality in older outpatients with T2D.

Keywords: sarcopenia, muscle mass, older, mortality, aged, diabetes 


\section{INTRODCUTION}

The number of people with type 2 diabetes mellitus (T2D), including older people, has been on the increase worldwide (1). In older people, sarcopenia, which is defined as age-related loss of muscle mass, strength, and function (2), is of grave concern. According to the Asian Working Group for Sarcopenia (2), sarcopenia is characterized by muscle mass loss and low muscle strength or low physical performance. T2D has reportedly been associated with a 1.55 -fold higher risk of sarcopenia in the older population than in the general population (3). Therefore, sarcopenia in people with T2D requires more attention than that in individuals without diabetes.

Sarcopenia has been associated with cardiometabolic diseases (CVD) (4-6), respiratory diseases (7), and low quality of life $(8,9)$. In addition, sarcopenia has reportedly been associated with an increased risk of mortality in the general population (1013). On the other hand, few studies have investigated the association between sarcopenia and mortality in people with T2D (14, 15). Previous studies have evaluated the effect of low muscle strength (14) or low muscle mass (15) on mortality but not the effect of sarcopenia on mortality. Thus, the purpose of this prospective cohort study was to investigate the effect of sarcopenia, including both muscle mass loss and low muscle strength, on mortality in people with T2D.

\section{MATERIALS AND METHODS}

\section{Study Design, Setting, and Participants}

This prospective cohort study was a part of the KAMOGAWADM cohort study, which has been conducted since 2014 (16). This cohort includes outpatients visiting the Department of Endocrinology and Metabolism, Kyoto Prefectural University of Medicine Hospital (Kyoto, Japan) and the Department of Diabetology, Kameoka Municipal Hospital (Kameoka, Japan). This study was approved by the local research ethics committee (No. RBMR-E-466-6) and was carried out in accordance with the ethical standards laid down in the 1964 Declaration of Helsinki and its later amendments. After obtaining written informed consent, medical data were anonymously gathered and compiled into a database. In this study, the data of all people with T2D were extracted from the database (17). Exclusion criteria were as follows: 1) no data on handgrip strength; 2) no data on body composition; 3) no data on potential covariates, such as medication, lifestyles, and metabolic parameters, including glycosylated hemoglobin Alc (HbAlc), creatinine, triglycerides, and blood pressure; 4) inaccurate data; and 5) people aged under 60 years (18).

\section{Data on Lifestyle Characteristics, Past Medical Histories, Medications, and Metabolic Parameters}

On analyzing body composition, the following data were also obtained:

Lifestyle characteristics, such as exercise, smoking, and alcohol, were evaluated. "Habit of exercise" was defined as performing any kind of physical activity at least once a week, "habit of smoking" as currently smoking cigarettes or another tobacco product, and "habit of alcohol" as daily alcohol consumption.

Past medical histories regarding CVD, including angina, coronary heart disease, heart failure, prior acute myocardial infarction, stroke (ischemic or hemorrhagic), and cancers, were obtained from electronic medical records.

Data on diabetes and hypertension medications as well as on usage of steroids were obtained from electronic medical records. Duration of diabetes at baseline was determined based on any one of the following: declaration from people, the date of the first abnormal laboratory report on diabetes, or the date of the first diabetes-related treatment, whichever preceded.

Laboratory data, including creatinine, HbAlc, and triglycerides, were obtained from venous blood samples following overnight fasting. HbA1c was measured using highperformance liquid chromatography and was expressed in National Glycohemoglobin Standardization Program units. The estimated glomerular filtration rate (eGFR; $\mathrm{mL} / \mathrm{min} / 1.73 \mathrm{~m}^{2}$ ) was calculated using the Japanese Society of Nephrology equation $\left(\mathrm{eGFR}=194 \times\right.$ serum creatinine $\mathrm{c}^{-1.094} \times \mathrm{age}^{-0.287} \times$ [0.739 for women]) (19).

Blood pressure measurement was performed automatically using an automatic blood pressure measurement device (HEM906; OMRON, Kyoto, Japan) in a quiet space after 5 min of rest. The handgrip strength of each hand was measured using a handgrip dynamometer (Smedley, Takei Scientific Instruments Co., Ltd., Niigata, Japan), and the maximum value was used for analysis (20).

Body composition was evaluated using a multifrequency impedance body composition analyzer, InBody 720 (InBody Japan, Tokyo, Japan), which has been reported to have good correlation with dual-energy X-ray absorptiometry (21). Body weight (BW, $\mathrm{kg}$ ), appendicular muscle mass $(\mathrm{kg})$, and body fat mass $(\mathrm{kg})$ were obtained. Body mass index $\left(\mathrm{BMI}, \mathrm{kg} / \mathrm{m}^{2}\right)$ and skeletal muscle mass index $\left(\mathrm{SMI}, \mathrm{kg} / \mathrm{m}^{2}\right)$ were calculated as follows: BW $(\mathrm{kg}) \div$ height squared $\left(\mathrm{m}^{2}\right)$ and appendicular muscle mass $(\mathrm{kg}) \div$ height squared $\left(\mathrm{m}^{2}\right)$, respectively.

\section{Definitions of Sarcopenia}

Sarcopenia was defined based on the Asian Working Group for Sarcopenia guidelines, using handgrip strength and SMI (2). The cutoff values for handgrip strength indicating low muscle strength were $<28 \mathrm{~kg}$ for men and $<18 \mathrm{~kg}$ for women, and those indicating a low SMI were $<7.0 \mathrm{~kg} / \mathrm{m}^{2}$ for men and $<5.7 \mathrm{~kg} /$ $\mathrm{m}^{2}$ for women. People who had both low muscle strength and low SMI were diagnosed with sarcopenia (2).

\section{Outcome of This Study and Follow-Up}

The outcome of this study was the occurrence of all-cause mortality. Death events were obtained from medical records. The follow-up duration (month) was calculated from the date of examination at baseline to the time of death, date of last followup (for people who ceased hospital visits), date of transfer to other hospitals, or otherwise, up to January 2021. 


\section{Statistical Analysis}

Statistical analyses were performed using EZR (Saitama Medical Center, Jichi Medical University, Saitama, Japan) (22), which is a graphical user interface for R (The R Foundation for Statistical Computing, Vienna, Austria).

Mean (standard deviation) or frequencies of potential confounding variables were expressed. Participants were divided into two groups according to the presence or absence of sarcopenia. The differences in continuous and categorical values were evaluated using the Student's t-test and chi-square or Fisher's exact test, respectively. Correlations were analyzed using the Pearson's correlation coefficient.

Kaplan-Meier analysis was performed to obtain a graphical representation of time against incident all-cause mortality, and a log-rank test was performed to assess the difference between participants with and without sarcopenia.

Due to the low incidence of all-cause mortality for statistical analysis, we used propensity scores to preserve statistical power by reducing the covariates into a single variable. To assess the propensity score, the dependent variable was the presence of sarcopenia. Propensity scores were evaluated using multivariable logistic regression models that included the following parameters: age, sex, duration of diabetes, history of heart disease, history of cancer, smoking, exercise, alcohol consumption, sodium-glucose cotransporter-2 (SGLT2) inhibitor, glucagon-like peptide-1 (GLP-1) receptor agonist, insulin, corticosteroid, hypertension, BMI, HbAlc, triglycerides, and creatinine. The C-statistic for the propensity score model was 0.89 , which displays acceptable discrimination. Thereafter, the hazard ratio (HR) for the risk of incident all-cause mortality in the presence of sarcopenia was calculated using a Cox regression model.

Subsequently, we investigated the effect of low SMI or low muscle strength on incident all-cause mortality using KaplanMeier analyses and the Cox regression model.

\section{RESULTS}

In this study, 702 people with T2D were recruited from the database, and 306 were excluded (Figure 1). Thus, 396 people were included in this study.

Among the 396 participants (232 men and 164 women), the average age and duration of diabetes were 71.3 (6.3) years and 16.3 (11.3) years, respectively (Table 1). The proportions of low muscle strength, low SMI, and sarcopenia were 26.8\%, 28.3\%, and $14.6 \%$, respectively. During the average 40.5 (16.5) months of follow-up, 13 people died (cancers, 7; infections, 3; CVD, 2; and unknown, 1).

SMI was negatively correlated with age $(r=-0.207, p<0.001)$ and duration of diabetes $(r=-0.106, p=0.036)$. Handgrip strength was negatively correlated with age $(r=-0.286$, $p<0.001)$ and duration of diabetes $(r=-0.141, p=0.005)$. BMI was positively associated with SMI $(r=0.512, p<0.001)$ and handgrip strength $(r=0.103, p=0.040)$. On the other hand, hemoglobin A1c was not correlated with both SMI $(r=-0.077$, $p=0.127)$ and handgrip strength $(r=0.002, p=0.963)$ (Table 2).

Figure 2 shows results of the Kaplan-Meier curve for cumulative survival rate according to the presence or absence of sarcopenia. The presence of sarcopenia was associated with higher mortality risk (log-rank test, $p<0.001)$. Sarcopenia was also associated with allcause mortality after adjusting for covariates (HR, 6.12; $95 \%$ confidence interval [CI], 1.52-24.7; $p=0.011$ ) (Table 3).

Figure 3 shows the results of the Kaplan-Meier survival curve for cumulative survival rate according to the presence or absence of low muscle strength or low SMI. The presence of sarcopenia, low muscle strength, and low SMI were associated with a higher mortality risk (both log-rank test, $p<0.001$ ). In addition, both low muscle strength and low SMI were associated with all-cause mortality after adjusting for covariates (low muscle strength: HR, 8.76; 95\% CI, 2.16-35.5), $p=0.002$; and low SMI: HR, 4.14; 95\% CI, 1.08-15.9, $p=0.039$ ) (Table 2).

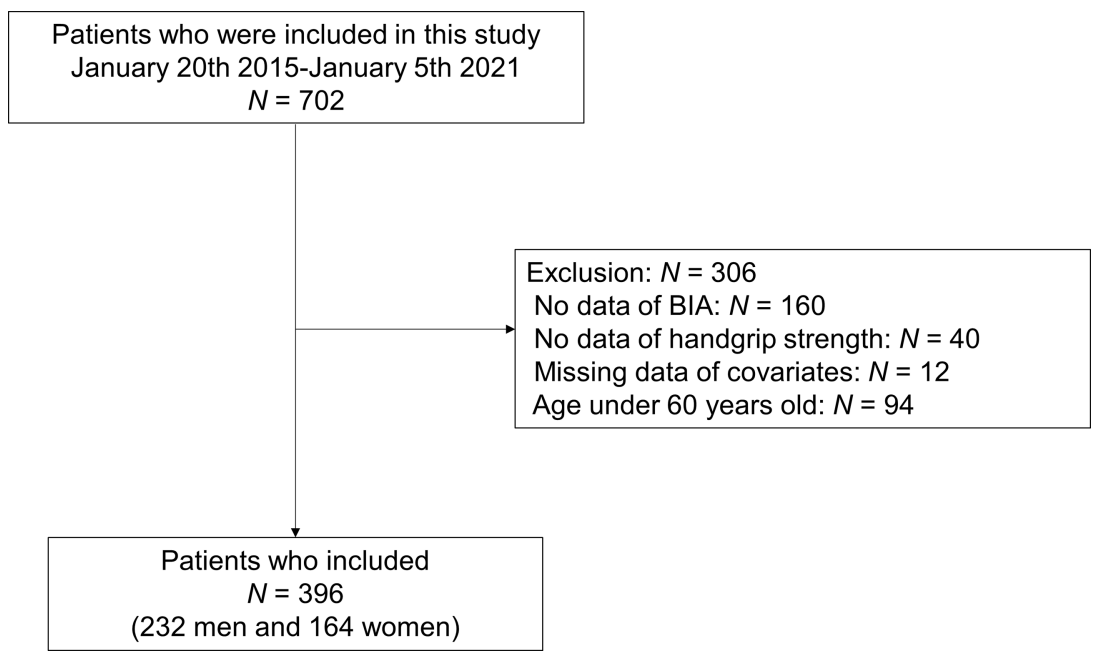

FIGURE 1 | Study flow diagram for the registration of participants. BIA, bioimpedance analysis. 
TABLE 1 | Clinical characteristics of study participants with and without sarcopenia.

\begin{tabular}{|c|c|c|c|c|}
\hline & All $N=396$ & Sarcopenia (-) N = 338 & Sarcopenia (+) N = 58 & $p$ \\
\hline Sex (men/women) & 232/164 & $195 / 143$ & $37 / 21$ & 0.476 \\
\hline Age (years) & $71.3(6.3)$ & $70.3(5.8)$ & $77.3(5.7)$ & $<0.001$ \\
\hline Duration of diabetes (years) & $16.3(11.3)$ & $15.4(11.0)$ & $21.4(11.9)$ & $<0.001$ \\
\hline Family history of diabetes (-/+) & 238/158 & $198 / 140$ & 40.18 & 0.178 \\
\hline Height (cm) & $160.6(8.7)$ & $161.1(8.7)$ & $157.7(8.3)$ & 0.005 \\
\hline Body weight (kg) & $61.1(10.8)$ & $62.6(10.5)$ & $52.2(8.4)$ & $<0.001$ \\
\hline Body mass index $\left(\mathrm{kg} / \mathrm{m}^{2}\right)$ & $23.6(3.7)$ & $24.1(3.6)$ & $20.9(2.6)$ & $<0.001$ \\
\hline Systolic blood pressure (mmHg) & 133.5 (18.3) & $134.1(18.3)$ & $130.4(18.3)$ & 0.164 \\
\hline Diastolic blood pressure $(\mathrm{mmHg})$ & $75.4(11.8)$ & $76.6(11.2)$ & $68.5(13.1)$ & $<0.001$ \\
\hline Antihypertensive drugs (-/+) & $166 / 230$ & $144 / 194$ & $22 / 36$ & 0.673 \\
\hline Presence of hypertension $(-/+)$ & $121 / 275$ & $104 / 234$ & $17 / 41$ & 0.945 \\
\hline SGLT2 inhibitor (-/+) & $339 / 57$ & $286 / 52$ & $53 / 5$ & 0.249 \\
\hline GLP-1 receptor agonist (-/+) & $342 / 54$ & $292 / 46$ & 50.8 & 1.000 \\
\hline Insulin (-/+) & 295/101 & $254 / 84$ & $41 / 17$ & 0.578 \\
\hline Corticosteroids (-/+) & $382 / 14$ & $330 / 8$ & $52 / 6$ & 0.009 \\
\hline History of heart disease $(-/+)$ & $320 / 76$ & $283 / 55$ & $37 / 21$ & $<0.001$ \\
\hline History of cancer $(-/+)$ & $331 / 65$ & $283 / 55$ & $48 / 10$ & 1.000 \\
\hline Habit of smoking $(-/+)$ & $337 / 59$ & $286 / 52$ & $51 / 7$ & 0.649 \\
\hline Habit of exercise $(-/+)$ & 198/198 & $175 / 163$ & $23 / 35$ & 0.118 \\
\hline Habit of drinking alcohol (-/+) & $277 / 119$ & 236/102 & $41 / 17$ & 1.000 \\
\hline Hemoglobin A1c (\%) & $7.3(1.0)$ & $7.2(1.1)$ & $7.4(1.0)$ & 0.294 \\
\hline Hemoglobin A1c (mmol/mol) & $55.9(11.5)$ & $55.6(11.5)$ & $57.3(10.9)$ & 0.294 \\
\hline Plasma glucose (mmol/L) & $147.7(47.3)$ & $8.1(2.7)$ & $8.5(2.5)$ & 0.310 \\
\hline Creatinine $(\mu \mathrm{mol} / \mathrm{L})$ & $76.3(33.7)$ & 76.3 (33.9) & $76.6(33.0)$ & 0.943 \\
\hline eGFR $\left(\mathrm{mL} / \mathrm{min} / 1.73 \mathrm{~m}^{2}\right)$ & $66.3(18.3)$ & $66.3(18.1)$ & 66.3 (19.9) & 0.986 \\
\hline Triglycerides (mmol/L) & $1.4(0.9)$ & $1.5(0.9)$ & $1.3(0.6)$ & 0.143 \\
\hline HDL cholesterol (mmol/L) & $1.6(0.5)$ & $1.6(0.4)$ & $1.5(0.5)$ & 0.701 \\
\hline Handgrip strength (kg) & $27.1(8.7)$ & $28.4(8.5)$ & $19.7(6.1)$ & $<0.001$ \\
\hline Low muscle strength $(-/+)$ & 290/106 & $290 / 48$ & $0 / 58$ & $<0.001$ \\
\hline Appendicular muscle mass (kg) & $17.9(3.9)$ & $18.4(3.8)$ & $14.9(3.2)$ & $<0.001$ \\
\hline Skeletal muscle mass $\left(\mathrm{kg} / \mathrm{m}^{2}\right)$ & $6.9(1.0)$ & $7.0(0.9)$ & $5.9(0.8)$ & $<0.001$ \\
\hline Low skeletal muscle mass (-/+) & $284 / 112$ & $284 / 54$ & $0 / 58$ & $<0.001$ \\
\hline Incident mortality $(-/+)$ & $383 / 13$ & $332 / 6$ & $51 / 7$ & $<0.001$ \\
\hline 1000 patient years of follow-up & 1.34 & 1.17 & 0.17 & $<0.001$ \\
\hline Incident mortality/ 1000 patient years of follow-up & 9.72 & 5.14 & 41.3 & $<0.001$ \\
\hline
\end{tabular}

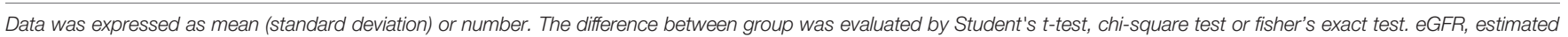
glomerular filtration rate; and HDL, high-density lipoprotein.

TABLE 2 | The correlation between the variables of diabetes and skeletal muscle mass or handgrip strength.

\begin{tabular}{|c|c|c|c|c|}
\hline & \multicolumn{2}{|c|}{ Skeletal muscle mass $\left(\mathrm{kg} / \mathrm{m}^{2}\right)$} & \multicolumn{2}{|c|}{ Handgrip strength (kg) } \\
\hline & $r$ & $p$ & $r$ & $p$ \\
\hline Age (years) & -0.207 & $<0.001$ & -0.286 & $<0.001$ \\
\hline Duration of diabetes (years) & -0.106 & 0.036 & -0.141 & 0.005 \\
\hline Body mass index (kg/m2) & 0.512 & $<0.001$ & 0.103 & 0.040 \\
\hline Hemoglobin A1c (\%) & -0.077 & 0.127 & 0.002 & 0.963 \\
\hline Hemoglobin A1c (mmol/mol) & -0.077 & 0.127 & 0.002 & 0.963 \\
\hline Plasma glucose (mmol/L) & -0.087 & 0.086 & -0.037 & 0.461 \\
\hline
\end{tabular}

Correlations were analyzed using the Pearson's correlation coefficient.

\section{DISCUSSION}

The main finding of this study was that sarcopenia is associated with incident all-cause mortality in older outpatients with T2D after adjusting for covariates. In addition, sub-analysis revealed that both low handgrip strength and low SMI were also associated with incident all-cause mortality in older outpatients with T2D, a finding similar to that of previous studies $(14,15)$. The association between low muscle strength (14) or low muscle mass (15) and mortality in old people with T2D has been reported; however, there are no studies reporting an association between sarcopenia, defined by both low muscle strength and low muscle mass, and mortality in old people with T2D.

Previous meta-analyses have revealed that sarcopenia is a risk factor for morality not only in community-dwelling older individuals $(11,23)$ but also in older nursing-home residents $(24)$. 


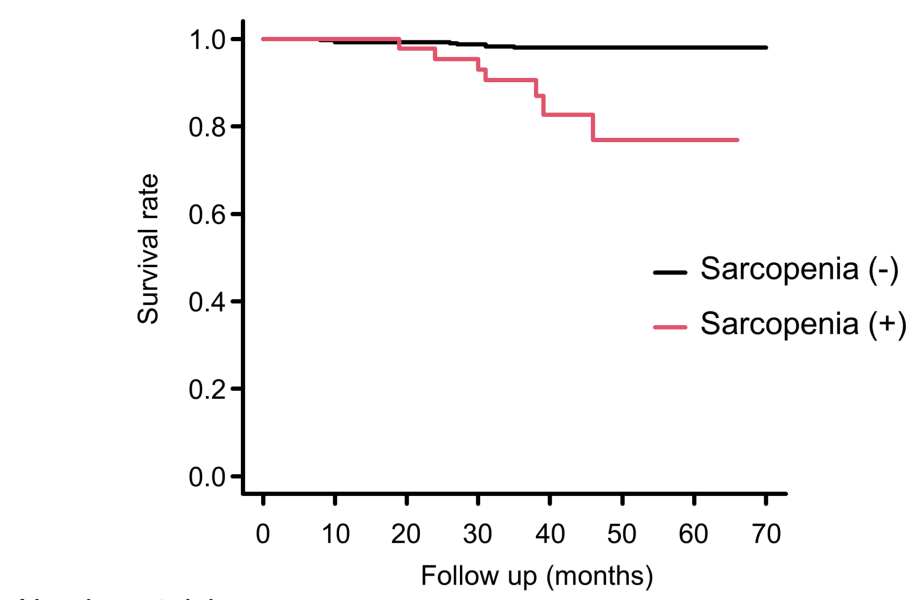

Number at risk

$\begin{array}{lcccccccc}\text { Sarcopenia (-) } & 338 & 306 & 297 & 277 & 217 & 145 & 6 & 1 \\ \text { Sarcopenia }(+) & 58 & 52 & 43 & 40 & 19 & 12 & 1 & 0\end{array}$

FIGURE 2 | Kaplan-Meier survival curve according to the presence or absence of sarcopenia. According to the log-rank test, $p<0.001$.

TABLE 3 | Hazard ratio of the presence or absence of sarcopenia, low muscle strength or low skeletal muscle mass for the all-cause mortality.

\begin{tabular}{|c|c|c|c|c|c|}
\hline & & \multicolumn{2}{|c|}{ Model 1} & \multicolumn{2}{|c|}{ Model 2} \\
\hline Sarcopenia & $\begin{array}{l}(-) \\
(+)\end{array}$ & $\begin{array}{c}\text { Ref } \\
8.86(2.96-26.5)\end{array}$ & $\begin{array}{c}- \\
<0.001\end{array}$ & $\begin{array}{c}\text { Ref } \\
6.12(1.52-24.7)\end{array}$ & $\begin{array}{c}- \\
0.011\end{array}$ \\
\hline Low muscle strength & $\begin{array}{l}(-) \\
(+)\end{array}$ & $\begin{array}{c}\text { Ref } \\
11.5(3.16-41.9)\end{array}$ & $\begin{array}{c}- \\
<0.001\end{array}$ & $\begin{array}{c}\text { Ref } \\
8.76(2.16-35.5)\end{array}$ & $\begin{array}{c}- \\
0.002\end{array}$ \\
\hline Low skeletal muscle mass & $\begin{array}{l}(-) \\
(+)\end{array}$ & $\begin{array}{c}\text { Ref } \\
6.38(1.96-20.7)\end{array}$ & $\begin{array}{c}- \\
0.002\end{array}$ & $\begin{array}{c}\text { Ref } \\
4.14(1.08-15.9)\end{array}$ & $\begin{array}{c}- \\
0.039\end{array}$ \\
\hline
\end{tabular}

Since the cases of mortality is not enough. Propensity score was used for covariates. Propensity score was evaluated by multivariable logistic regression models that include the age, sex, duration of diabetes, history of heart disease, history of cancer, smoking, exercise, drinking alcohol, SGLT2 inhibitor, GLP-1 receptor agonist, insulin, corticosteroid, hypertension, body mass index, hemoglobin A1c, triglycerides, and creatinine. The sarcopenia for propensity score model was 0.89. Model 1 was unadjusted. Model 2 was adjusted for the propensity score.
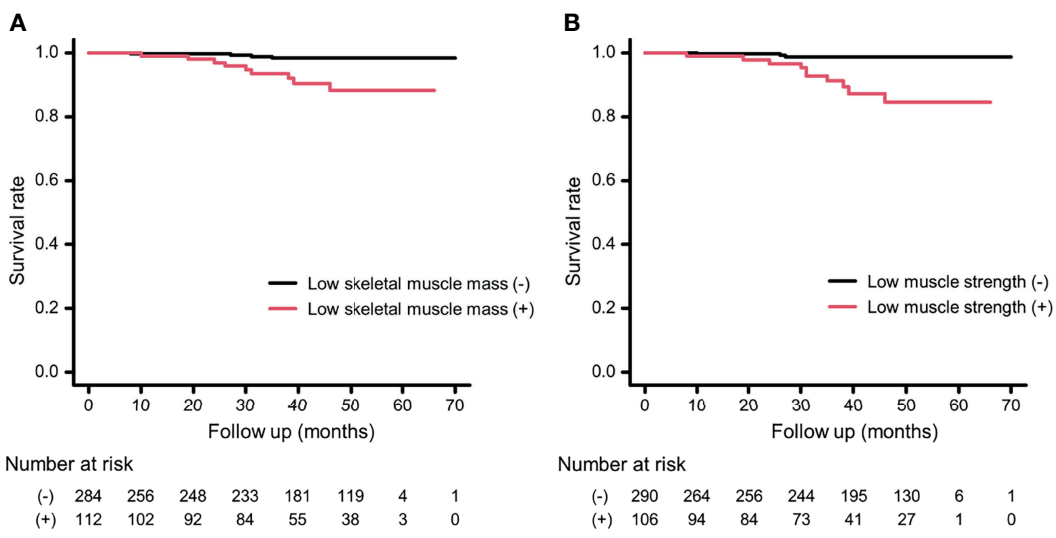

Number at risk

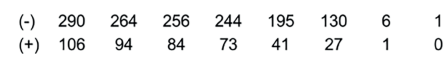

FIGURE 3 | Kaplan-Meier survival curve according to the presence or absence of low skeletal muscle mass or low muscle strength. (A) The presence or absence of low skeletal muscle mass. (B) The presence or absence of low muscle strength. According to the log-rank test, both $p<0.001$. 
Moreover, recent meta-analyses have revealed that sarcopenia is a mortality risk in people with colorectal cancer (25). In addition to these meta-analyses, recent studies have confirmed that sarcopenia is a risk factor for morality in people on hemodialysis (5) and in older hospitalized people (12). However, there are no reports investigating the association between sarcopenia, defined by both low muscle strength and low muscle mass, and mortality in people with T2D. These results were consistent with those from our study.

The possible mechanisms behind the association between sarcopenia and mortality risk are as follows. Sarcopenia has reportedly been linked to decreasing protein synthesis and increasing protein degradation, through increased oxidative stress (26), decreasing antioxidant defenses (26), and inflammations (27), leading to mortality (28). These factors are also associated with atherosclerosis. In fact, sarcopenia, especially low muscle strength, has been associated with blood pressure variability (29) and risk of cardiovascular events (4-6). In addition, sarcopenia has reportedly been associated with a high risk of malnutrition $(30,31)$, which aggravates sarcopenia, resulting in an increased mortality rate. Furthermore, although we adjusted for cancer as a covariate in this study, cachexia, characterized by systemic inflammation and abnormal metabolism with loss of muscle mass as well as reduced food intake, is often accompanied by sarcopenia in people with cancer (18). Thus, sarcopenia in older people with cancer has been shown to be predictive of a negative clinical outcome, due not only to the loss of tissue mass but also the loss of cell integrity $(32,33)$.

The treatment and prevention of sarcopenia is an important issue in T2D because the prevalence of sarcopenia has been reported to be higher in T2D than in the general population (3). In fact, among our study participants, the prevalence of sarcopenia was $15.1 \%$, which was almost similar to that of previous studies $(34,35)$. Exercise training as well as adequate protein and calorie intakes are necessary to maintain and increase muscle mass (36-40). Furthermore, handgrip exercises decrease vascular resistance by a cholinergic mechanism (41), although it is possible that such a physiological effect may be attenuated in people with T2D because of autonomic nervous dysfunction. In addition, good glycemic control (35) and proper usage of medications, including insulin (35), GLP-1 receptor agonist (42, 43), and SGLT2 inhibitor $(44,45)$, have been reported to improve sarcopenia.

The limitations of this study should not go unmentioned. First, we used the multifrequency impedance body composition analyzer for body composition analysis, although dual-energy X-ray absorptiometry is the gold standard method for evaluating skeletal muscle mass. A previous study reported that bioimpedance analysis overestimated fat mass and underestimated lean soft tissue mass when validated against dual-energy X-ray absorptiometry (21). Second, the incidence of all-cause mortality was relatively small. Thus, we could not perform a detailed analysis of the cause of death. The number of outcomes was relatively small, further follow-up is

\section{REFERENCES}

1. Bradley D, Hsueh W. Type 2 Diabetes in the Elderly: Challenges in a Unique Patient Population. J Geriatr Med Gerontol (2016) 2(2):14. doi: 10.23937/ 2469-5858/1510014 imperative. Third, vitamin D status, including serum vitamin D level and vitamin D intake, did not assess in this study, although vitamin $\mathrm{D}$ status was reported to be associated with muscle mass loss (46) and muscle mass strength (47). Fourth, this study included only people with $\mathrm{T} 2 \mathrm{D}$; generalization to the other groups is hence uncertain. Finally, this study only included Japanese individuals. Therefore, it is possible that the results of this study may not be generalized to individuals from other backgrounds.

In conclusion, sarcopenia is associated with incident all-cause mortality in older outpatients with T2D. The present findings contribute to our understanding of the importance of sarcopenia in preventing future mortality in older people with T2D.

\section{DATA AVAILABILITY STATEMENT}

The datasets used and/or analyzed during the current study are available from the corresponding author on reasonable request.

\section{ETHICS STATEMENT}

The studies involving human participants were reviewed and approved by Kyoto prefectural University of Medicine. The patients/participants provided their written informed consent to participate in this study.

\section{AUTHOR CONTRIBUTIONS}

FT obtained, analyzed, and interpreted data and wrote the manuscript. YH planned and designed the work, obtained, analyzed, and interpreted data, and wrote the manuscript. AK, $\mathrm{RS}$, and TO obtained and interpreted data and contributed to the discussion. NK, HO, NN, SM, and TS obtained data and contributed to the discussion. EU and $\mathrm{MH}$ planned the work, obtained data, and contributed to the discussion. MA and MY obtained data and contributed to the discussion. MF planned and designed the work, obtained and interpreted data, and contributed to the discussion. All authors have checked the final version, and agree to be responsible for the work to ensure that any questions related to the accuracy or completeness of any of the work are appropriately investigated and resolved.

\section{ACKNOWLEDGMENTS}

We would like to thank Editage (www.editage.com) for English language editing.

2. Chen LK, Woo J, Assantachai P, Auyeung TW, Chou MY, Iijima K, et al. Asian Working Group for Sarcopenia: 2019 Consensus Update on Sarcopenia Diagnosis and Treatment. J Am Med Dir Assoc (2020) 21(3):300-307.e2. doi: $10.1016 /$ j.jamda.2019.12.012

3. Anagnostis P, Gkekas NK, Achilla C, Pananastasiou G, Taouxidou P, Mitsiou $\mathrm{M}$, et al. Type 2 Diabetes Mellitus Is Associated With Increased Risk of 
Sarcopenia: A Systematic Review and Meta-Analysis. Calcif Tissue Int (2020) 107(5):453-63. doi: 10.1007/s00223-020-00742-y

4. Kang DO, Park SY, Choi BG, Na JO, Choi CU, Kim EJ, et al. Prognostic Impact of Low Skeletal Muscle Mass on Major Adverse Cardiovascular Events in Coronary Artery Disease: A Propensity Score-Matched Analysis of a Single Center All-Comer Cohort. J Clin Med (2019) 8(5):712. doi: 10.3390/ jcm8050712

5. Lee YH, Kim JS, Jung SW, Hwang HS, Moon JY, Jeong KH, et al. Gait Speed and Handgrip Strength as Predictors of All-Cause Mortality and Cardiovascular Events in Hemodialysis Patients. BMC Nephrol (2020) 21 (1):166. doi: 10.1186/s12882-020-01831-8

6. Harada K, Suzuki S, Ishii H, Aoki T, Hirayama K, Shibata Y, et al. Impact of Skeletal Muscle Mass on Long-Term Adverse Cardiovascular Outcomes in Patients With Chronic Kidney Disease. Am J Cardiol (2017) 119(8):1275-80. doi: 10.1016/j.amjcard.2017.01.003

7. Bone AE, Hepgul N, Kon S, Maddocks M. Sarcopenia and Frailty in Chronic Respiratory Disease: Lessons from Gerontology. Chron Respir Dis (2017) 14 (1):85-99. doi: 10.1177/1479972316679664

8. Beaudart C, Biver E, Reginster JY, Rizzoli R, Rolland Y, Bautmans I, et al. Validation of the SarQoL ${ }^{\circledR}$, a Specific Health-Related Quality of Life Questionnaire for Sarcopenia. J Cachexia Sarcopenia Muscle (2017) 8: (2):238-44. doi: 10.1002/jcsm.12149

9. Tsekoura M, Kastrinis A, Katsoulaki M, Billis E, Gliatis J. Sarcopenia and Its Impact on Quality of Life. Adv Exp Med Biol (2017) 987:213-8. doi: 10.1007/ 978-3-319-57379-3_19

10. Brown JC, Harhay MO, Harhay MN. Sarcopenia and Mortality Among a Population-Based Sample of Community-Dwelling Older Adults. J Cachexia Sarcopenia Muscle (2016) 7(3):290-8. doi: 10.1002/jcsm.12073

11. Beaudart $C$, Zaaria $M$, Pasleau $F$, Reginster JY, Bruyère $O$. Health Outcomes of Sarcopenia: A Systematic Review and Meta-Analysis. PloS One (2017) 12(1): e0169548. doi: 10.1371/journal.pone.0169548

12. Scheerman K, Meskers CGM, Verlaan S, Maier AB. Sarcopenia, Low Handgrip Strength, and Low Absolute Muscle Mass Predict Long-Term Mortality in Older Hospitalized Patients: An Observational Inception Cohort Study. J Am Med Dir Assoc (2021) 22(4):816-820.e2. doi: 10.1016/ j.jamda.2020.12.016

13. Sobestiansky S, Michaelsson K, Cederholm T. Sarcopenia Prevalence and Associations With Mortality and Hospitalisation by Various Sarcopenia Definitions in 85-89 Year Old Community-Dwelling Men: A Report From the ULSAM Study. BMC Geriatr (2019) 19(1):318. doi: 10.1186/s12877-019-1338-1

14. Hamasaki H, Kawashima Y, Katsuyama H, Sako A, Goto A, Yanai H. Association of Handgrip Strength With Hospitalization, Cardiovascular Events, and Mortality in Japanese Patients With Type 2 Diabetes. Sci Rep (2017) 7(1):7041. doi: 10.1038/s41598-017-07438-8

15. Miyake H, Kanazawa I, Tanaka KI, Sugimoto T. Low Skeletal Muscle Mass is Associated With the Risk of All-Cause Mortality in Patients With Type 2 Diabetes Mellitus. Ther Adv Endocrinol Metab (2019) 10:2042018819842971. doi: $10.1177 / 2042018819842971$

16. Sakai R, Hashimoto Y, Ushigome E, Miki A, Okamura T, Matsugasumi M, et al. Late-Night-Dinner Is Associated With Poor Glycemic Control in People With Type 2 Diabetes: The KAMOGAWA-DM Cohort Study. Endocr J (2018) 65(4):395-402. doi: 10.1507/endocrj.EJ17-0414

17. American Diabetes Association. 2. Classification and Diagnosis of Diabetes. Diabetes Care (2016) 39 Suppl 1:S13-22. doi: 10.2337/dc16-S005

18. Shafiee G, Keshtkar A, Soltani A, Ahadi Z, Larijani B, Heshmat R. Prevalence of Sarcopenia in the World: A Systematic Review and Meta- Analysis of General Population Studies. J Diabetes Metab Disord (2017) 16:21. doi: 10.1186/s40200-017-0302-x

19. Matsuo S, Imai E, Horio M, Yasuda Y, Tomita K, Nitta K, et al. Revised Equations for Estimated GFR From Serum Creatinine in Japan. Am J Kidney Dis (2009) 53(6):982-92. doi: 10.1053/j.ajkd.2008.12.034

20. Kaji A, Hashimoto Y, Kobayashi Y, Sakai R, Okamura T, Miki A, et al. Sarcopenia Is Associated With Tongue Pressure in Older Patients With Type 2 Diabetes: A Cross-Sectional Study of the KAMOGAWA-DM Cohort Study. Geriatr Gerontol Int (2019) 19(2):153-8. doi: 10.1111/ggi.13577

21. Kim M, Shinkai S, Murayama H, Mori S. Comparison of Segmental Multifrequency Bioelectrical Impedance Analysis With Dual-Energy X-Ray Absorptiometry for the Assessment of Body Composition in a Community-
Dwelling Older Population. Geriatr Gerontol Int (2015) 15(8):1013-22. doi: $10.1111 /$ ggi. 12384

22. Kanda Y. Investigation of the Freely Available Easy-to-Use Software 'Ezr' for Medical Statistics. Bone Marrow Transplant (2013) 48(3):452-8. doi: 10.1038/ bmt.2012.244

23. Liu P, Hao Q, Hai S, Wang H, Cao L, Dong B. Sarcopenia as a Predictor of AllCause Mortality Among Community-Dwelling Older People: A Systematic Review and Meta-Analysis. Maturitas (2017) 103:16-22. doi: 10.1016/ j.maturitas.2017.04.007

24. Zhang X, Wang C, Dou Q, Zhang W, Yang Y, Xie X. Sarcopenia as a Predictor of All-Cause Mortality Among Older Nursing Home Residents: A Systematic Review and Meta-Analysis. BMJ Open (2018) 8(11):e021252. doi: 10.1136/ bmjopen-2017-021252

25. Trejo-Avila M, Bozada-Gutiérrez K, Valenzuela-Salazar C, Herrera-Esquivel J, Moreno-Portillo M. Sarcopenia Predicts Worse Postoperative Outcomes and Decreased Survival Rates in Patients With Colorectal Cancer: A Systematic Review and Meta-Analysis. Int J Colorectal Dis (2021) 36(6):1077-96. doi: 10.1007/s00384-021-03839-4

26. Marzetti E, Lees HA, Wohlgemuth SE, Leeuwenburgh C. Sarcopenia of Aging: Underlying Cellular Mechanisms and Protection by Calorie Restriction. Biofactors (2009) 35(1):28-35. doi: 10.1002/biof.5

27. Westbury LD, Fuggle NR, Syddall HE, Duggal NA, Shaw SC, Maslin K, et al. Relationships Between Markers of Inflammation and Muscle Mass, Strength and Function: Findings From the Hertfordshire Cohort Study. Calcif Tissue Int (2018) 102(3):287-95. doi: 10.1007/s00223-017-0354-4

28. Yoshihara F. Systemic Inflammation Is a Key Factor for Mortality Risk Stratification in Chronic Kidney Disease Patients With Coronary Artery Calcification. Circ J (2016) 80(7):1537-8. doi: 10.1253/circj.CJ16-0506

29. Hashimoto Y, Kaji A, Sakai R, Hamaguchi M, Okada H, Ushigome E, et al. Sarcopenia Is Associated With Blood Pressure Variability in Older Patients With Type 2 Diabetes: A Cross-Sectional Study of the KAMOGAWA-DM Cohort Study. Geriatr Gerontol Int (2018) 18(9):1345-9. doi: 10.1111/ ggi.13487

30. Tamura Y, Omura T, Toyoshima K, Araki A. Nutrition Management in Older Adults With Diabetes: A Review on the Importance of Shifting Prevention Strategies From Metabolic Syndrome to Frailty. Nutrients (2020) 12(11):3367. doi: $10.3390 /$ nu12113367

31. Okamura T, Miki A, Hashimoto Y, Kaji A, Sakai R, Osaka T, et al. Shortage of Energy Intake Rather Than Protein Intake Is Associated With Sarcopenia in Elderly Patients With Type 2 Diabetes: A Cross-Sectional Study of the KAMOGAWA-DM Cohort. J Diabetes (2019) 11(6):477-83. doi: 10.1111/ 1753-0407.12874

32. Norman K, Wirth R, Neubauer M, Eckardt R, Stobäus N. The Bioimpedance Phase Angle Predicts Low Muscle Strength, Impaired Quality of Life, and Increased Mortality in Old Patients With Cancer. J Am Med Dir Assoc (2015) 16(2):173.e17-173.e1.73E22. doi: 10.1016/j.jamda.2014.10.024

33. Otten L, Stobäus N, Franz K, Genton L, Müller-Werdan U, Wirth R, et al. Impact of Sarcopenia on 1-Year Mortality in Older Patients With Cancer. Age Ageing (2019) 48(3):413-8. doi: 10.1093/ageing/afy212

34. Izzo A, Massimino E, Riccardi G, Della Pepa G. A Narrative Review on Sarcopenia in Type 2 Diabetes Mellitus: Prevalence and Associated Factors. Nutrients (2021) 13(1):183. doi: 10.3390/nu13010183

35. Sugimoto K, Ikegami H, Takata Y, Katsuya T, Fukuda M, Akasaka H, et al. Glycemic Control and Insulin Improve Muscle Mass and Gait Speed in Type 2 Diabetes: The MUSCLES-DM Study. J Am Med Dir Assoc (2021) 22(4):834838.e1. doi: 10.1016/j.jamda.2020.11.003

36. Kimura T, Okamura T, Iwai K, Hashimoto Y, Senmaru T, Ushigome E, et al. Japanese Radio Calisthenics Prevents the Reduction of Skeletal Muscle Mass Volume in People With Type 2 Diabetes. BMJ Open Diabetes Res Care (2020) 8(1):e001027. doi: 10.1136/bmjdrc-2019-001027

37. Hashimoto Y, Kaji A, Sakai R, Takahashi F, Kawano R, Hamaguchi M, et al. Effect of Exercise Habit on Skeletal Muscle Mass Varies With Protein Intake in Elderly Patients With Type 2 Diabetes: A Retrospective Cohort Study. Nutrients (2020) 12(10):3220. doi: 10.3390/nu12103220

38. Das SK, Roberts SB, Bhapkar MV, Villareal DT, Fontana L, Martin CK, et al. Body-Composition Changes in the Comprehensive Assessment of Long-Term Effects of Reducing Intake of Energy (CALERIE)-2 Study: A 2-Y Randomized 
Controlled Trial of Calorie Restriction in Nonobese Humans. Am J Clin Nutr (2017) 105(4):913-27. doi: 10.3945/ajcn.116.137232

39. Wu PY, Huang KS, Chen KM, Chou CP, Tu YK. Exercise, Nutrition, and Combined Exercise and Nutrition in Older Adults With Sarcopenia: A Systematic Review and Network Meta-Analysis. Maturitas (2021) 145:3848. doi: 10.1016/j.maturitas.2020.12.009

40. Kawano R, Takahashi F, Hashimoto Y, Okamura T, Miki A, Kaji A, et al. Short Energy Intake is Associated With Muscle Mass Loss in Older Patients With Type 2 Diabetes: A Prospective Study of the KAMOGAWA-DM Cohort. Clin Nutr (2021) 40(4):1613-20. doi: 10.1016/j.clnu.2021.02.049

41. Vianna LC, Fadel PJ, Secher NH, Fisher JP. A Cholinergic Contribution to the Circulatory Responses Evoked at the Onset of Handgrip Exercise in Humans. Am J Physiol Regul Integr Comp Physiol (2015) 308(7):R597-604. doi: 10.1152/ ajpregu.00236.2014

42. Hong Y, Lee JH, Jeong KW, Choi CS, Jun HS. Amelioration of Muscle Wasting by Glucagon-Like Peptide-1 Receptor Agonist in Muscle Atrophy. J Cachexia Sarcopenia Muscle (2019) 10(4):903-18. doi: 10.1002/jcsm.12434

43. Gurjar AA, Kushwaha S, Chattopadhyay S, Das N, Pal S, China SP, et al. Long Acting GLP-1 Analog Liraglutide Ameliorates Skeletal Muscle Atrophy in Rodents. Metabolism (2020) 103:154044. doi: 10.1016/j.metabol.2019.154044

44. Sano M, Meguro S, Kawai T, Suzuki Y. Increased Grip Strength With SodiumGlucose Cotransporter 2. J Diabetes (2016) 8(5):736-7. doi: 10.1111/1753-0407.12402

45. Okamura T, Hashimoto Y, Osaka T, Fukuda T, Hamaguchi M, Fukui M. The Sodium-Glucose Cotransporter 2 Inhibitor Luseogliflozin can Suppress Muscle Atrophy in Db/Db Mice by Suppressing the Expression of Foxo1. J Clin Biochem Nutr (2019) 65(1):23-8. doi: 10.3164/jcbn.18-114

46. Takahashi F, Hashimoto Y, Kaji A, Sakai R, Kawate Y, Okamura T, et al. Vitamin Intake and Loss of Muscle Mass in Older People With Type 2 Diabetes: A Prospective Study of the KAMOGAWA-DM Cohort. Nutrients (2021) 13(7):2335. doi: 10.3390/nu13072335

47. Visser M, Deeg DJH, Lips P. Low Vitamin D and High Parathyroid Hormone Levels as Determinants of Loss of Muscle Strength and Muscle Mass (Sarcopenia): The Longitudinal Aging Study Amsterdam. J Clin Endocrinol Metab (2003) 88:5766-72. doi: 10.1210/jc.2003-030604

Conflict of Interest: YH reports personal fees from Novo Nordisk Pharma Ltd., Daiichi Sankyo Co. Ltd., Sanofi K.K., Mitsubishi Tanabe Pharma Corp., Ono Pharma Co., Ltd., Takeda Pharma Co., Ltd., and Sumitomo Dainippon Pharma Co. Ltd. EU received personal fees from MSD K.K., Mitsubishi Tanabe Pharma Corp., AstraZeneca plc, Daiichi Sankyo Co. Ltd., Novo Nordisk Pharma Ltd., Taisho Toyama Pharma Co., Ltd., Kowa Pharma Co. Ltd., Takeda Pharma Co., Ltd., Kyowa Kirin Co. Ltd., Sumitomo Dainippon Pharma Co. Ltd., Astellas Pharma Inc., and Nippon Boehringer Ingelheim Co. Ltd. outside the submitted work and received grant support from the Astellas Foundation for Research on Metabolic Disorders and the Japanese Study Group for Physiology and Management of Blood Pressure, donated fund Laboratory of Diabetes therapeutics is an endowment department, supported with an unrestricted grant from Ono Pharma. Co., Ltd. MH received grants from Daiichi Sankyo Co. Ltd., Astellas Pharma Inc., Mitsubishi Tanabe Pharma Corp., Novo Nordisk Pharma Ltd., Nippon Boehringer Ingelheim Co. Ltd., Sanofi K.K., Takeda Pharma Co. Ltd, Sumitomo Dainippon Pharma Co. Ltd., Asahi Kasei Pharma, Kyowa Kirin Co. Ltd., and Eli Lilly Japan K.K., outside the submitted work. MA received personal fees from Takeda Pharmaceutical Co., Ltd., Abbott Japan Co., Ltd., Sumitomo Dainippon Pharma Co., Ltd., Kowa Pharmaceutical Co., Ltd., Novo Nordisk Pharma Ltd., Ono Pharmaceutical Co., Ltd., AstraZeneca K.K., and Chugai Pharmaceutical Co., Ltd., outside the submitted work. MY received personal fees from Ono Phama Co., Ltd., Kowa Pharma Co. Ltd., AstraZeneca plc., Sumitomo Dainippon Pharma Co. Ltd., Kyowa Kirin Co. Ltd., MSD K.K., Takeda Pharma Co. Ltd, Kowa Pharma Co. Ltd., and Daiichi Sankyo Co. Ltd. outside the submitted work. MF received grants from Taisho Pharma Co., Ltd., Mitsubishi Tanabe Pharma Corp, Novo Nordisk Pharma Ltd., Ono Pharma Co. Ltd., Kowa Pharma Co. Ltd., Sanofi K.K., Nippon Boehringer Ingelheim Co. Ltd., Daiichi Sankyo Co. Ltd., Kissei Phama Co. Ltd., MSD K.K., Kyowa Kirin Co., Ltd., Sumitomo Dainippon Pharma Co., Ltd., Eli Lilly Japan K.K., Tejin Pharma Ltd., Takeda Pharma Co. Ltd., Nippon Chemiphar Co., Ltd., Astellas Pharma Inc., Abbott Japan Co. Ltd., Sanwa Kagagu Kenkyusho CO., LtD., Johnson \& Johnson k.k. Medical Co., and Terumo Corp., and received honoraria from AstraZeneca K.K., Taisho Pharma Co., Ltd., Ono Pharma Co. Ltd., Novo Nordisk Pharma Ltd., Sanofi K.K., Teijin Pharma Ltd., Takeda Pharma Co. Ltd., Astellas Pharma Inc., MSD K.K., Mitsubishi Tanabe Pharma Corp., Eli Lilly Japan K.K., Kissei Pharma Co., Ltd., Sumitomo Dainippon Pharma Co. Ltd., Daiichi Sankyo Co. Ltd., Mochida Pharma Co. Ltd., Kowa Pharma Co. Ltd., Arkray Inc., Abbott Japan Co. Ltd., Sanwa Kagaku Kenkyusho Co. Ltd., Kyowa Kirin Co. Ltd., Nippon Boehringer Ingelheim Co., Ltd., Medtronic Japan Co. Ltd., Bayer Yakuhin, Ltd., and Nipro Corp. outside the submitted work.

The remaining authors declare that the research was conducted in the absence of any commercial or financial relationships that could be construed as a potential conflict of interest.

Publisher's Note: All claims expressed in this article are solely those of the authors and do not necessarily represent those of their affiliated organizations, or those of the publisher, the editors and the reviewers. Any product that may be evaluated in this article, or claim that may be made by its manufacturer, is not guaranteed or endorsed by the publisher.

Copyright (๑) 2021 Takahashi, Hashimoto, Kaji, Sakai, Okamura, Kitagawa, Okada, Nakanishi, Majima, Senmaru, Ushigome, Hamaguchi, Asano, Yamazaki and Fukui. This is an open-access article distributed under the terms of the Creative Commons Attribution License (CC BY). The use, distribution or reproduction in other forums is permitted, provided the original author(s) and the copyright owner(s) are credited and that the original publication in this journal is cited, in accordance with accepted academic practice. No use, distribution or reproduction is permitted which does not comply with these terms. 